\title{
Thermodynamics and Statistical Mechanics of frozen systems in inherent states
}

\author{
Annalisa Fierro, Mario Nicodemi, 7 and Antonio Coniglio \\ Dipartimento di Fisica, Università di Napoli "Federico II", INFM, Unità di Napoli, \\ Complesso Universitario Monte Sant'Angelo, Via Cinthia, I-80126, Napoli, Italy
}

(Dated: November 8, 2018)

\begin{abstract}
We discuss a Statistical Mechanics approach in the manner of Edwards to the "inherent states" (defined as the stable configurations in the potential energy landscape) of glassy systems and granular materials. We show that at stationarity the inherent states are distributed according a generalized Gibbs measure obtained assuming the validity of the principle of maximum entropy, under suitable constraints. In particular we consider three lattice models (a diluted Spin Glass, a monodisperse hard-sphere system under gravity and a hard-sphere binary mixture under gravity) undergoing a schematic "tap dynamics", showing via Monte Carlo calculations that the time average of macroscopic quantities over the tap dynamics and over such a generalized distribution coincide. We also discuss about the general validity of this approach to non thermal systems.
\end{abstract}

\section{INTRODUCTION}

There are many complex systems where thermal fluctuations are small enough that the temperature of the external bath, $T_{\text {bath }}$, can be considered zero. Examples are supercooled liquids quenched at zero temperature in metastable states (blocked configurations), called "inherent structures", which correspond to the local minima of the potential energy in the particles configuration space [1, 2, 3, 4]. Granular materials [5] at rest are another important example of system frozen [6] in mechanically stable microstates (blocked configurations), which by analogy with the glass terminology can also be called inherent states.

The issue we consider here, which recently raised considerable interest, is to investigate the possibility to describe these systems by using concepts from Statistical Mechanics, as Edwards [7] suggested for granular media more than ten years ago. His assumption was that, by gently shaking the system under the constrain of fixed volume $V$, the distribution over the mechanically stable (blocked) states would be uniform. This leads to the definition of a configurational entropy, $S=\ln \Omega$, where $\Omega$ is the number of mechanically stable states corresponding to the fixed volume $V$ and energy $E$, and to the concept of compactivity, $X^{-1}=\frac{\partial \ln \Omega}{\partial V}$. In a similar way one can also define a configurational temperature, $T_{\text {conf }}^{-1} \equiv \beta_{\text {conf }}=\frac{\partial \ln \Omega}{\partial E}$.

Also in glasses, following for example the inherent structure approach [1, 2, 3, 4, one can define a configurational entropy associated to the number of inherent structures corresponding to a fixed energy, $E$, and consequently the configurational temperature. When the system is frozen at zero temperature in one of his inherent states it does not evolve anymore. However, one can explore the inherent structures space essentially in two ways. One way is by quenching the system over and over

\footnotetext{
*Also at Department of Mathematics, Imperial College, London,
} SW7 2BZ, U.K. from an equilibrium temperature, T, to zero temperature 11, 3, 4. Using this procedure, Sciortino et al. 3] found that in a supercooled glass forming liquid, studied by molecular dynamics simulations, the configurational temperature numerically coincides with the equilibrium temperature $T$, provided that $T$ is low enough.

Another way to visit the inherent structures is by letting the system aging in contact with an almost zero bath temperature, $T_{\text {bath }}$. During the aging process an effective temperature, $T_{d y n}$, can be defined via the offequilibrium extension of the Fluctuation-Dissipation ratio [8]. It happens that in mean field models [9] this effective temperature coincides with the above configurational temperature. The possibility to introduce an effective temperature for granular media via the extension of the Fluctuation-Dissipation relation, was suggested in Ref. [10].

The connection between Edwards approach for granular media and the results in glass theory has been pointed out in 10, 11, 12 and 13, 14. In particular in Ref. 11] it was shown that, for a class of finite dimensional systems, in the limit $T_{\text {bath }} \rightarrow 0, T_{d y n}$ coincides in fact with the configurational temperature, predicted by the Edwards' hypothesis.

In Ref. [13, 14] the inherent states are visited in an other way by using a tap dynamics (i.e., a procedure similar to that used in the compaction of real granular materials), where each tap consists in raising the bath temperature to a value $T_{\Gamma}$ and, after a lapse of time $\tau_{0}$, quenching it back to zero. By cyclically repeating the process the system explores the space of the inherent states [13, 14, 15, 16, 17, 18, 19, 20]. Once the stationary state is reached one can define a temperature, $T_{f d}$, via the equilibrium Fluctuation-Dissipation relation. One can then see that, if Edwards' assumption applies, $T_{f d}$ coincides with the configurational temperature. This has been verified in fact for different finite dimensional models 13, 14, 15. It was also shown numerically that for low enough $T_{\Gamma}$ one has that $T_{f d}=T_{\text {conf }} \simeq T_{\Gamma}$, confirming on lattice models for granular media the result of Ref. [3]. In fact when the duration of each single tap is infinite $\left(\tau_{0} \rightarrow \infty\right)$, the tap coincides with the way 
to explore the inherent states implemented in molecular dynamics simulations for Lennard Jones mixtures [1, 3. However the method used in [3] only allows the calculation of $T_{\text {conf }}$ when the configurational temperatures is low, i.e., where all the different temperatures almost coincide. Many other studies confirming Edwards' approach have also been presented [16, 17, 18, 21].

In this paper we give a comprehensive view of the results obtained in 13, 14, 15] by considering other models and giving more details. In particular we study here three schematic lattice models for glassy systems and granular media, i.e., a diluted Spin Glass, a monodisperse hardsphere system under gravity and a hard-sphere binary mixture under gravity. In particular, in the diluted Spin Glass and in the monodisperse hard-sphere system under gravity, the asymptotic states reached by the system are found to be described only by the configurational temperature. Whereas in the hard-sphere binary mixture under gravity the asymptotic states are found to be described by two thermodynamic parameters [22], coinciding with the two configurational temperatures which characterize the distribution among the inherent states when the principle of maximum entropy is satisfied under the constraint that the energies of the two species are independently fixed. In Ref. [15] a description of the segregation observed in the binary system in term of these two temperatures is also given.

In Sect.s II A and II B, the frustrated lattice gas model and the results of its study with the tap dynamics are respectively presented. In Sect. IID, the same results are obtained at higher density where the system at small temperature reaches a quasi-stationary state in which one time quantities decay as the logarithm of time. In Sect. IIC Edwards' hypothesis is formulated using the principle of maximum entropy. The results obtained in the monodisperse hard-sphere system under gravity are shown in Sect. III. In Sect. IV the Statistical Mechanics approach is extended to the hard-sphere binary mixture under gravity, where two thermodynamic parameters are necessary to describe the asymptotic states reached by the system. Finally, in the Conclusions we draw a picture of the Statistical Mechanics approach to systems found in inherent states, as it emerges from our extensive investigation.

\section{THE FRUSTRATED LATTICE GAS MODEL}

\section{A. The model}

Recently a lattice model has been introduced to describe glass formers 25, 26 and, in presence of gravity, granular materials [19, 27, 28. The Hamiltonian of the model is:

$$
-H=J \sum_{\langle i j\rangle}\left(\epsilon_{i j} S_{i} S_{j}-1\right) n_{i} n_{j}+\mu \sum_{i} n_{i}
$$

where the sum $\sum_{\langle i j\rangle}$ is over nearest neighbor sites, $S_{i}=$ \pm 1 are Ising spins, $n_{i}=0,1$ are occupation variables, $\mu$ is the particle chemical potential and $\epsilon_{i j}$ are quenched and random variables, equal to \pm 1 with equal probability. This model reproduces the Ising spin glass in the limit $\mu \rightarrow \infty$ (i.e., when all sites are occupied, $n_{i} \equiv 1$ ).

In the other limit, $J \rightarrow \infty$, the model describes a frustrated lattice gas with properties recalling those of a "frustrated" liquid. In fact the first term of Hamiltonian (1) implies that two nearest neighbor sites can be freely occupied only if their spin variables satisfy the interaction, that is if $\epsilon_{i j} S_{i} S_{j}=1$, otherwise they feel a strong repulsion. To make the connection with a liquid, we note that the internal degree of freedom, $S_{i}$, may represent for example the internal orientation of a non spherical particle. Two particles can be nearest neighbors only if the relative orientation is appropriate, otherwise they have to move apart. Since in a frustrated loop the spins cannot satisfy all interactions, in this model particle configurations in which a frustrated loop is fully occupied are not allowed. The frustrated loops in the model are the same of the spin glass model and correspond in the liquid to those loops which, due to geometrical hindrance, cannot be fully occupied by the particles. In $3 D$ [26, 29], the model has a maximum density $\rho_{\max } \simeq 0.68$, and a transition at $\rho_{c} \simeq 0.62$ where the non-linear spin susceptibility diverges.

In the present paper, the $3 D$ cubic frustrated lattice gas model with $J$ finite is considered. The value of particle density, $\rho=\sum_{i} n_{i} / L^{3}$ ( $L$ is the lattice linear size), is fixed, and a Monte Carlo tap dynamics, which allows the system to explore its inherent states, is applied. During the dynamics, the system cyclically evolves for a time $\tau_{0}$ (the tap duration 30]) at a finite value of the bath temperature, $T_{\Gamma}$ (the tap amplitude), and afterwards it is suddenly frozen at zero temperature in one of its inherent states (at zero temperature the system does not evolve anymore if the energy cannot be decreased by one single particle movement). After each tap, when the system is at rest, we record the quantities of interest. The time, $t$, considered is therefore discrete and coincides with the number of taps.

\section{B. The results obtained under the tap dynamics}

We first consider the case $\rho=0.65$ 31]. At this value of the density the system under the tap dynamics reaches a stationary state for each value of $T_{\Gamma}$ (and $\tau_{0}$ ) considered. In Fig.s 1 and 2 32, the self-scattering two time function, $F_{q}\left(t, t_{w}\right)=\sum_{i} e^{\left.q \cdot r_{i}(t)-\vec{r}_{i}\left(t_{w}\right)\right]} / \rho L^{3}$, and the energy, $E(t)$, of the inherent states, obtained for $T_{\Gamma}=0.3 \mathrm{~J}$ and $\tau_{0}=$ $1 M C S$, are shown. The curves, $F_{q}\left(t, t_{w}\right)$, for different $t_{w}$, collapse onto a single master function, when they are plotted as function of $t-t_{w}$, and the energy, $E(t)$, reaches its time independent asymptotic value, showing that the system has reached a stationary state (our data are averaged up to 32 noise realizations; $L=8$ and $q=$ 


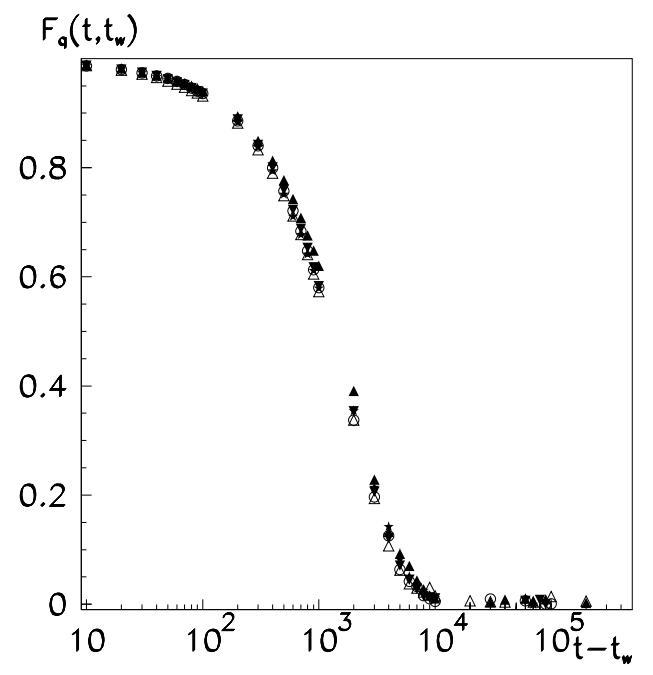

FIG. 1: The self-scattering two time function, $F_{q}\left(t, t_{w}\right)=$ $\sum_{i} e^{\vec{q} \cdot\left[\vec{r}_{i}(t)-\vec{r}_{i}\left(t_{w}\right)\right]} / \rho L^{3}$, with $q=\pi / 4$, as function of $t-t_{w}$ (for $t_{w}=10^{4}, 2 \cdot 10^{4}, 5 \cdot 10^{4}, 8 \cdot 10^{4}, 10^{5}$ ) in the frustrated lattice gas model for density $\rho=0.65$, during the tap dynamics, with tap amplitude $T_{\Gamma}=0.3 \mathrm{~J}$ and tap duration $\tau_{0}=1 M C S$. The function $F_{q}\left(t, t_{w}\right)$ only depends on $t-t_{w}$, showing that the system has reached stationarity.

$\pi / 4)$.

During the tap dynamics, in the stationary state, the time average of the energy, $\bar{E}$, and its fluctuations, $\overline{\Delta E^{2}}$, are calculated. In Fig.s 3 and $4, \bar{E}$ and $\overline{\Delta E^{2}}$ are shown as function the tap amplitude, $T_{\Gamma}$, (for several values of the tap duration, $\left.\tau_{0}\right)$. Apparently, $T_{\Gamma}$ is not the right thermodynamic parameter, since sequences of taps, with same $T_{\Gamma}$ and different $\tau_{0}$, give different values of $\bar{E}$ and $\overline{\Delta E^{2}}$. On the other hand, if the stationary states are indeed characterized by a single thermodynamic parameter, $\beta_{f d}$, the curves corresponding to different tap sequences (i.e. different $T_{\Gamma}$ and $\tau_{0}$ ) should collapse onto a single master function, when $\overline{\Delta E^{2}}$ is parametrically plotted as function of $\bar{E}$. This data collapse is in fact found and shown in Fig. 5. This is a prediction which could be easily checked in real granular materials (where one could consider the density which is easier to measure than the energy).

The thermodynamic parameter, $\beta_{f d}$, is defined apart from an integration constant, $\beta_{0}$, through the usual equilibrium Fluctuation-Dissipation relation:

$$
-\frac{\partial \bar{E}}{\partial \beta_{f d}}=\overline{\Delta E^{2}}
$$

By integrating eq. (2), $\beta_{f d}-\beta_{0}$ can be expressed as function of $\bar{E}$ or (for a fixed value of $\tau_{0}$ ) as function of $\beta_{\Gamma}: \beta_{f d}-\beta_{0} \equiv g\left(\beta_{\Gamma}\right)$. The constant $\beta_{0}$ is determined as explained in detail in the Appendix.

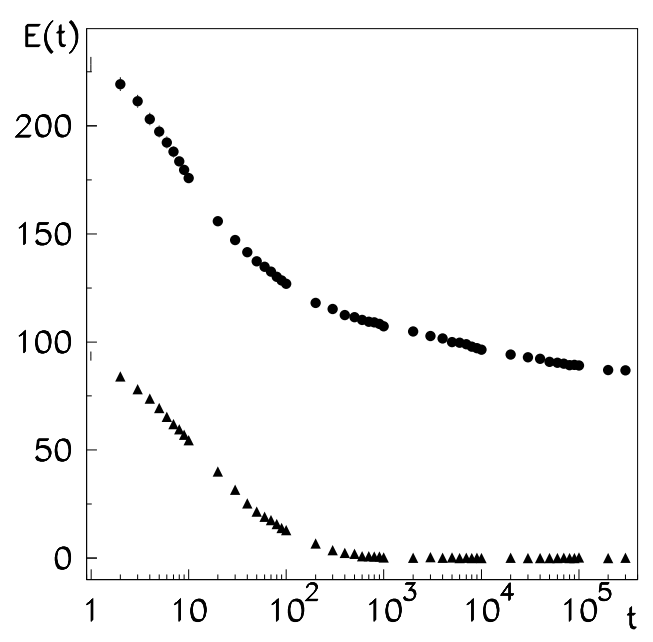

FIG. 2: The energy, $E(t)$, of the inherent states as function of the tap number $t$, in the frustrated lattice gas model during the tap dynamics with tap amplitude $T_{\Gamma}=0.3 \mathrm{~J}$ and tap duration $\tau_{0}=1 M C S$. The lower curve, corresponding to a density of particles $\rho=0.65$, exponentially saturates to its asymptotic value, whereas the upper curve, corresponding to $\rho=0.75$, shows a logarithmic relaxation at long times.

\section{The Edwards' averages}

In Sect. IIB we have found that the fluctuations of the energy in the stationary state depend only on the energy, $\bar{E}$, and not on the past history. If all macroscopic quantities depend only on the energy, $\bar{E}$, or on its conjugate thermodynamic parameter, $\beta_{f d}$, the stationary state can be genuinely considered a "thermodynamic state". In this case one can attempt to construct an equilibrium statistical mechanics, as originally suggested by Edwards [7].

More precisely one can try to find from basic general principles what is the probability distribution, $P_{r}$, of finding, in the stationary regime, the system in the inherent state $r$ of energy $E_{r}$ (see [13]). We assume that the distribution is given by the principle of maximum entropy, $S=-\sum_{r} P_{r} \ln P_{r}$, under the condition that the average energy is fixed: $E=\sum_{r} P_{r} E_{r}$. Thus, we have to maximize the following functional: $I\left[P_{r}\right]=-\sum_{r} P_{r} \ln P_{r}-\beta_{\text {conf }}\left(E-\sum_{r} P_{r} E_{r}\right)$. Here $\beta_{\text {conf }}$ is a Lagrange multiplier determined by the constraint on the energy and takes the name of "inverse configurational temperature". This procedure leads to the Gibbs result:

$$
P_{r}=\frac{e^{-\beta_{\text {conf } E_{r}}}}{Z}
$$

where $Z=\sum_{r} e^{-\beta_{\text {conf }} E_{r}}$. Using standard Statistical Mechanics it is easy to show that, in the thermodynamic limit, the entropy $S$ and $\beta_{\text {conf }}$ are also given by:

$$
S=\ln \Omega(E), \quad \beta_{\text {conf }}=\frac{\partial \ln \Omega}{\partial E}
$$




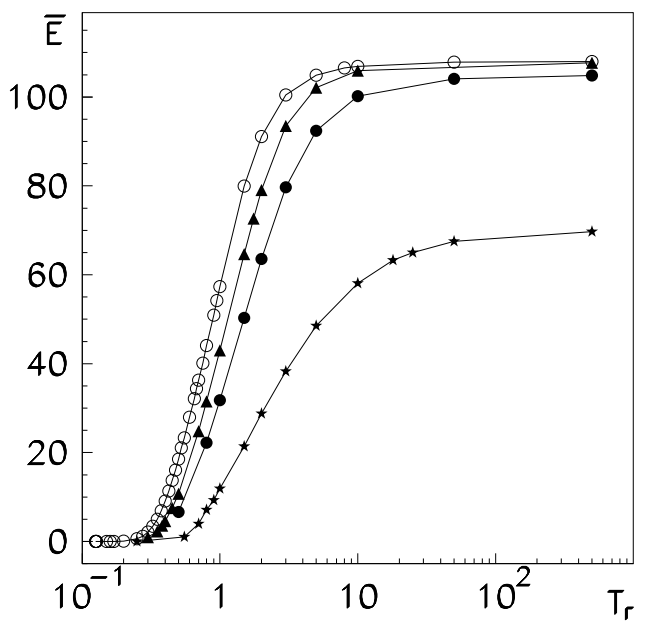

FIG. 3: The time average of the energy, $\bar{E}$, recorded in the stationary regime, as function of the tap amplitude, $T_{\Gamma}$ (in units of $J$ ), in the frustrated lattice gas model for $\rho=0.65$. The four different curves correspond to different values of the tap duration, $\tau_{0}=1,5,10, \infty M C S$ (from bottom to top). This shows that $T_{\Gamma}$ is not a right thermodynamic parameter, since sequences of taps with different $\tau_{0}$ give different values for the system observables.

where $\Omega(E)$ is the number of inherent states corresponding to energy $E$.

If the distribution in the stationary state coincides with eq.(33) the time average of the energy, $\bar{E}\left(\beta_{f d}\right)$, recorded during the taps sequences, must coincide with the ensemble average, $\langle E\rangle\left(\beta_{\text {conf }}\right)$, over the distribution eq.(3)). In order to check that we have independently calculated the average $\langle E\rangle$, as function of $\beta_{\text {conf }}$. We have simulated the model eq.(1) imposing that the only accessible states are the inherent states, as done in Ref. [11]. The only difference is that in the present paper the Edwards' averages are done in the canonical ensemble, whereas in Ref. [11] are done in the microcanonical ensemble. In particular we have constructed a new Hamiltonian, $\mathcal{H}^{\prime}\left(\left\{S_{i}, n_{i}\right\}\right)=\mathcal{H}\left(\left\{S_{i}, n_{i}\right\}\right)+\delta\left(\left\{S_{i}, n_{i}\right\}\right)$, by adding a term to eq.(1),$\delta\left(\left\{S_{i}, n_{i}\right\}\right)$, which is zero, if the configuration is an inherent state, and infinite, otherwise. The canonical distribution for this Hamiltonian gives a weight, $e^{-\beta_{\text {conf } f} \mathcal{H}^{\prime}}$, which coincides with the weight in the distribution of eq.(3), for each accessible configuration. Using the standard Monte Carlo simulations, we have calculated $\langle E\rangle\left(\beta_{\text {conf }}\right)$. Fig. 6 outlines a very good agreement between $\langle E\rangle\left(\beta_{\text {conf }}\right)$ and $\bar{E}\left(\beta_{f d}\right)$ (notice that there are no adjustable parameters).

Note that the maximum energy reached by the system under the tap dynamics, $E_{\max }\left(\tau_{0}\right) \equiv \bar{E}\left(T_{\Gamma} \rightarrow \infty, \tau_{0}\right)$, is less than the maximum energy of the inherent states, $\langle E\rangle\left(T_{\text {conf }} \rightarrow \infty\right)$, for every value of $\tau_{0}$ considered. Such a prediction, which may have important practical consequences (e.g., in powders technologies), is consistent

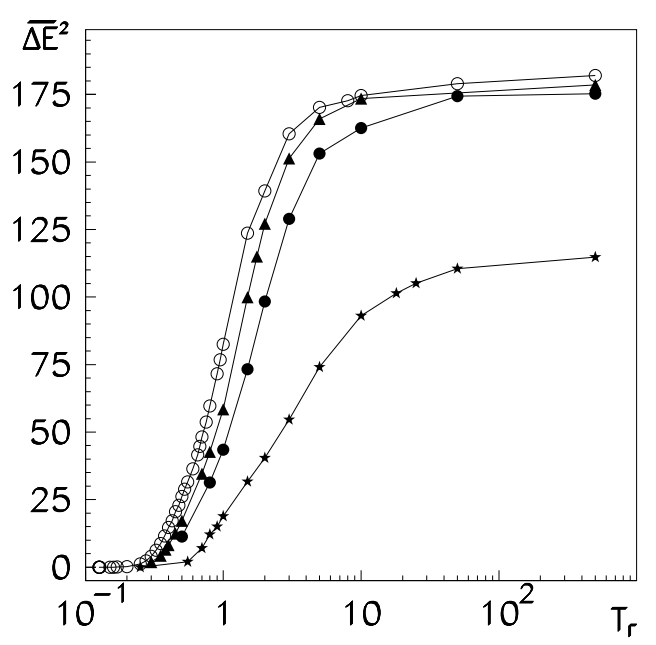

FIG. 4: The time average of energy fluctuations, $\overline{\Delta E^{2}}$, recorded in the stationary regime, as function of the tap amplitude, $T_{\Gamma}$ (in units of $J$ ), in the frustrated lattice gas model for $\rho=0.65$. The four different curves correspond to different values of the tap duration, $\tau_{0}=1,5,10, \infty M C S$ (from bottom to top). This shows again that $T_{\Gamma}$ is not a right thermodynamic parameter.

with some experimental observations on tapped granular materials [33], where the system density was shown to approach asymptotically a plateau value apparently higher than the minimal possible packing density (obtained, for instance, by just pouring grains in the container) even for very large tap amplitudes.

Using eq.(四), we have finally evaluated the configurational entropy as $S(E)-S_{0}=\int_{0}^{E} \beta_{\text {conf }}\left(E^{\prime}\right) d E^{\prime}$ (where the unknown non negative constant, $S_{0} \equiv S(E=0)$, is the entropy at $\left.T_{\text {conf }}=0\right)$. In Fig. 1, the configurational entropy, $S-S_{0}$, is plotted as function of $T_{\text {conf } f}$. We have also evaluated the integral $S^{\prime}(E)-S_{0}^{\prime} \equiv \int_{0}^{E} \beta_{f d}\left(E^{\prime}\right) d E^{\prime}$. In Fig. 7, $S^{\prime}-S_{0}^{\prime}$ is plotted as function of $T_{f d}$ and it is compared with the configurational entropy. The agreement is again very good.

\section{Quasi-stationary case}

We have also studied the frustrated lattice gas model for $\rho=0.75$. Differently from the previous case, for small enough values of the tap amplitude $T_{\Gamma}$, the system does not reach a stationary state during our observation time. In Fig. 2, the energy, $E(t)$, of the inherent states obtained for $T_{\Gamma}=0.3 J$ and $\tau_{0}=1 M C S$ is shown. $E(t)$ now changes in time and the system is not in a stationary state; however, $E(t)$, at long times decays very slowly [34. In this regime the time averages are computed over a time interval such that the energy is practically constant (in the case of Fig. 2, the time average is performed over the time interval $\left.\left(3 \cdot 10^{5}, 3 \cdot 10^{5}+10^{4}\right)\right)$. Performing 


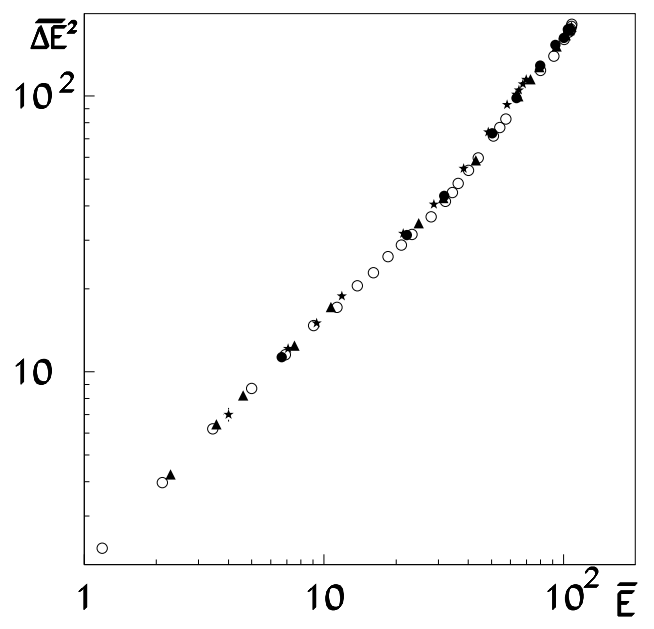

FIG. 5: The time averages of energy fluctuations, $\overline{\Delta E^{2}}$, when plotted as function of the time average of energy, $\bar{E}$, collapse onto a single master function, for all the different values of tap amplitude and duration, $T_{\Gamma}$ and $\tau_{0}$, plotted in Fig. 3. This shows that the system stationary states are indeed characterized by a single thermodynamic parameter, since the curves corresponding to different tap sequences (i.e. different $T_{\Gamma}$ and $\left.\tau_{0}\right)$ collapse on a "universal" function, when $\overline{\Delta E^{2}}$ is parametrically plotted as function of $\bar{E}$.

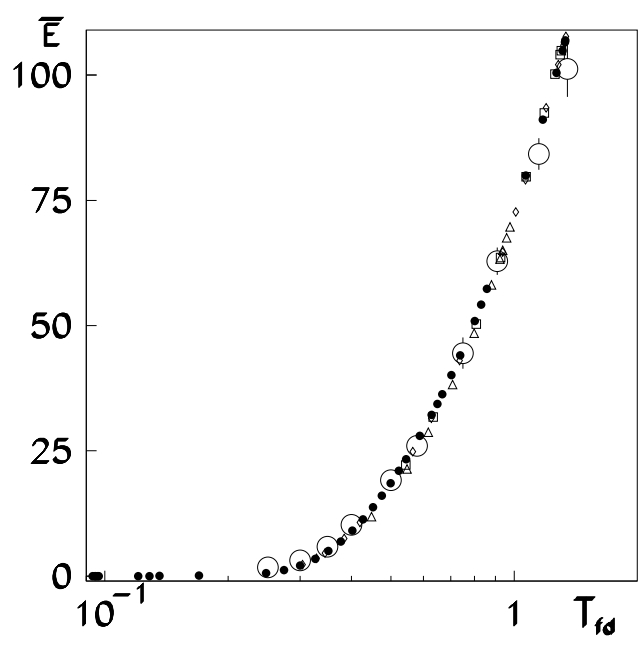

FIG. 6: The time average, $\bar{E}$, calculated in the stationary regime of the tap dynamics and the ensemble average over the Edwards' distribution eq.(3), $\langle E\rangle$ (the black empty circles), are plotted respectively as a function of $T_{f d}$ and $T_{\text {conf }}$ (in units of $J$ ), in the frustrated lattice gas model, at $\rho=0.65$. The two independently calculated sets of points show a very good agreement, outlining the success of Edwards' approach to describe the system macroscopic properties.

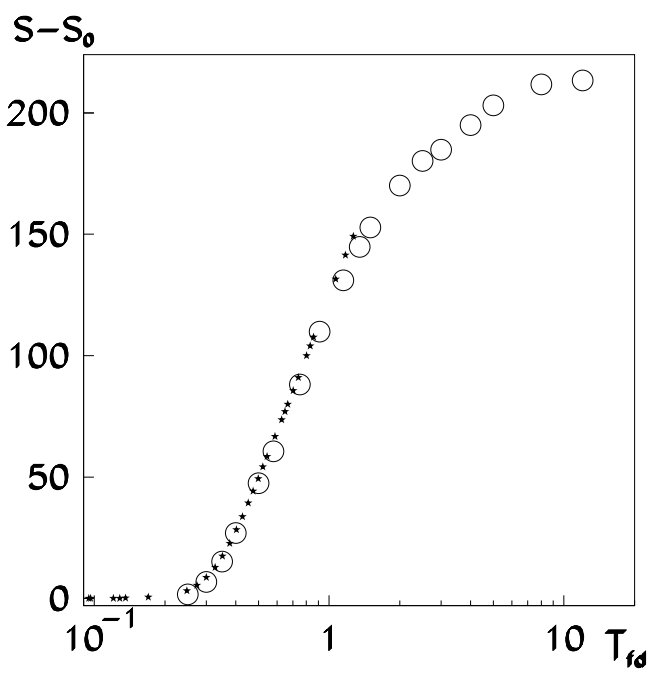

FIG. 7: The configurational entropy, $S-S_{0}$, (the black empty circles in figure) as function of $T_{\text {conf }}$ (in units of $J$ ), compared with $S^{\prime}(E)-S_{0}^{\prime} \equiv \int_{0}^{E} \beta_{f d}\left(E^{\prime}\right) d E^{\prime}$ plotted as function of $T_{f d}$ (in units of $J$ ), in the frustrated lattice gas model for $\rho=$ 0.65 . The unknown non negative constant $S_{0}$ is the entropy at $T_{\text {conf }}=0$.

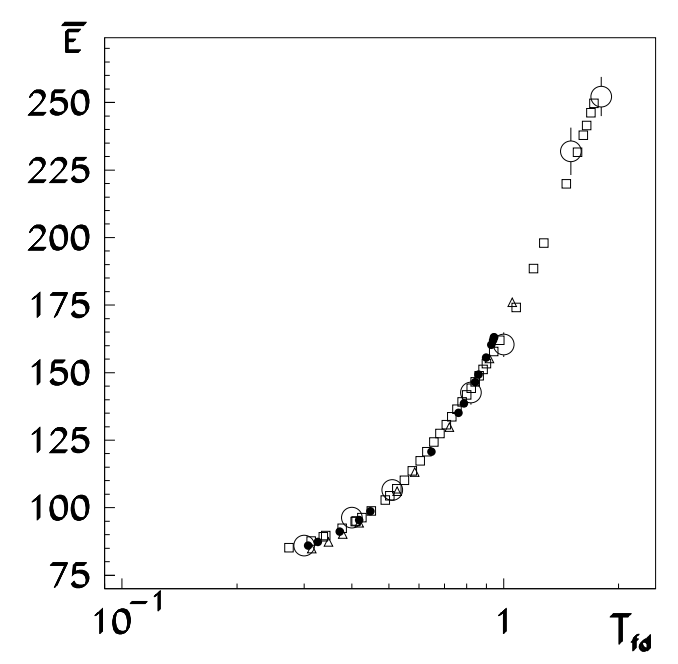

FIG. 8: The time average, $\bar{E}$, and the ensemble average over the distribution eq.(3), $\langle E\rangle$ (the black empty circles), plotted respectively as a function of $T_{f d}$ and $T_{\text {conf }}$ (in units of $J$ ), in the frustrated lattice gas model, at $\rho=0.75$. As well as at $\rho=0.65$, there is a very good agreement between the two independently calculated sets of points.

the same procedure described in the stationary case, a collapse of data is again found (see Fig.8).

We have again evaluated the configurational entropy, $S(E)-S_{0}=\int_{E_{\text {min }}}^{E} \beta_{\text {conf }}\left(E^{\prime}\right) d E^{\prime}$ (where the unknown non negative constant, $S_{0} \equiv S\left(E_{\min }\right)$, is the entropy at $T_{\text {conf }}=0$ and $E_{\min }$ is the minimum value of energy obtained [35]). In Fig. 9, the configurational entropy, 


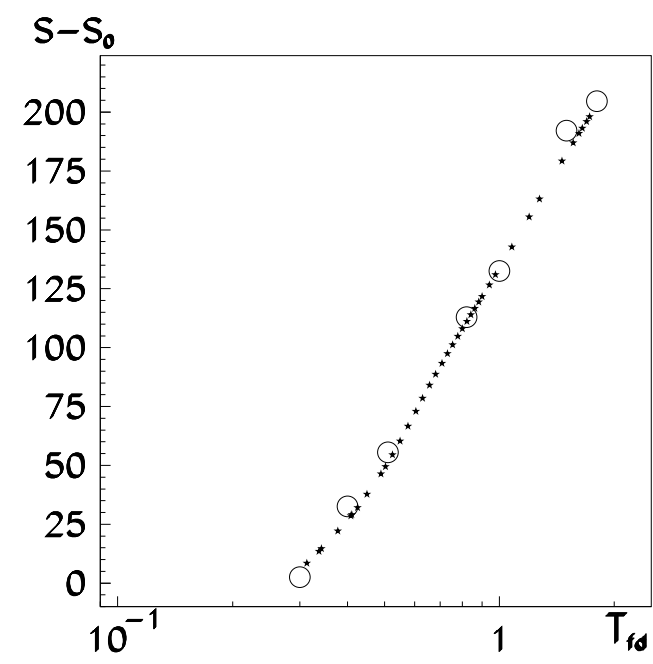

FIG. 9: The configurational entropy, $S-S_{0}$, (the black empty circles in figure) as function of $T_{\text {conf }}$ (in units of $J$ ), compared with $S^{\prime}(E)-S_{0}^{\prime} \equiv \int_{0}^{E} \beta_{f d}\left(E^{\prime}\right) d E^{\prime}$ plotted as function of $T_{f d}$ (in units of $J$ ), in the frustrated lattice gas model for $\rho=$ 0.75 . The unknown non negative constant $S_{0}$ is the entropy at $T_{\text {conf }}=0$.

$S-S_{0}$, is plotted as function of $T_{\text {conf }}$. We have also evaluated the integral $S^{\prime}(E)-S_{0}^{\prime} \equiv \int_{0}^{E} \beta_{f d}\left(E^{\prime}\right) d E^{\prime}$. In Fig. 9, $S^{\prime}-S_{0}^{\prime}$ is plotted as function of $T_{f d}$ and it is compared with the configurational entropy. The agreement is again very good.

We cannot exclude that the agreement here found even for low energy may be due to the fact that the system, which is not in a stationary state, is however very near stationarity.

\section{A MONODISPERSE HARD-SPHERE SYSTEM UNDER GRAVITY}

As a model more appropriate for granular media, we have also studied a system of monodisperse hard-sphere (with diameter $a_{0}=1$ ) under gravity, where the centers of mass of grains are constrained to move on the sites of a cubic lattice (see upper inset in Fig. 11). The Hamiltonian of the system is:

$$
\mathcal{H}=\mathcal{H}_{h c}\left(\left\{n_{i}\right\}\right)+g m \sum_{i} n_{i} z_{i}
$$

where the height of site $i$ is $z_{i}, g=1$ is the gravity acceleration, $m=1$ the grains mass, $n_{i}$ is the usual occupancy variable and $\mathcal{H}_{h c}\left(\left\{n_{i}\right\}\right)$ is the hard core term preventing the overlapping of nearest neighbors grains (the analogy with eq.(11) can be appreciated by writing down $\mathcal{H}_{h c}$ : it can be written as $\mathcal{H}_{h c}\left(\left\{n_{i}\right\}\right)=J \sum_{\langle i j\rangle} n_{i} n_{j}$, where the limit $J \rightarrow \infty$ is taken).

We have considered a system of $N=240$ particles, and performed a tap dynamics which allows the system

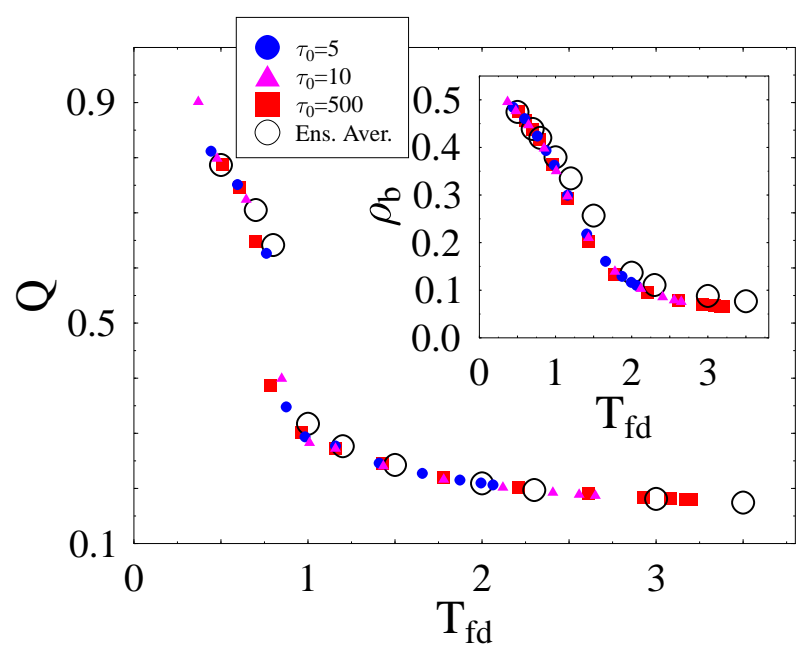

FIG. 10: The density self-overlap function, $Q$, and (upper inset) the system density on the bottom layer, $\rho_{b}$, plotted as function of $T_{f d}$ (in units $m g a_{0}$ ), compared with the ensemble averages over the distribution eq.(3) (the black empty circles), plotted as function of $T_{\text {conf }}$ (in units $m g a_{0}$ ), in the 3D monodisperse hard-sphere system under gravity. Also for this system there is a very good agreement between the independently calculated time averages over the tap dynamics and the statistical mechanics ensemble averages á la Edwards.

to explore its inherent states. We have considered 3 different values of the tap duration, $\tau_{0}=500,10,5 M C S$. In this case we again obtain that the asymptotic states reached by the system can be described by a single thermodynamic parameter, $\beta_{f d}$, evaluated by integration of eq. (2). We have moreover calculated the system density on the bottom layer, $\rho_{b}$, and the density self-overlap function, $Q$, and verified that, when plotted as function of $\beta_{f d}$, they scale on a single master function (see Fig. 10).

As described in Sect. IIC, we have calculated the Edwards' averages as function of $\beta_{\text {conf }}$. As we can see in Fig.11, we obtain a very good agreement between $\langle E\rangle\left(\beta_{\text {conf }}\right)$ and $\bar{E}\left(\beta_{f d}\right)$. The same agreement is found for the other quoted observables, $\rho_{b}$ and $Q$ (see Fig. 10).

\section{A HARD-SPHERE BINARY MIXTURE UNDER GRAVITY}

Finally we consider a hard-sphere binary system made of two species 1 (small) and 2 (large) with grain diameters $a_{0}$ and $\sqrt{2} a_{0}$, under gravity on a cubic lattice of spacing $a_{0}=1$. We set the units such that the two kinds of grain have masses $m_{1}=1$ and $m_{2}=2$, and the gravity acceleration is $g=1$. The hard core potential $\mathcal{H}_{h c}$ is such that two large nearest neighbor particles cannot overlap. This implies that only couples of small particles can be nearest neighbors on the lattice. The system 


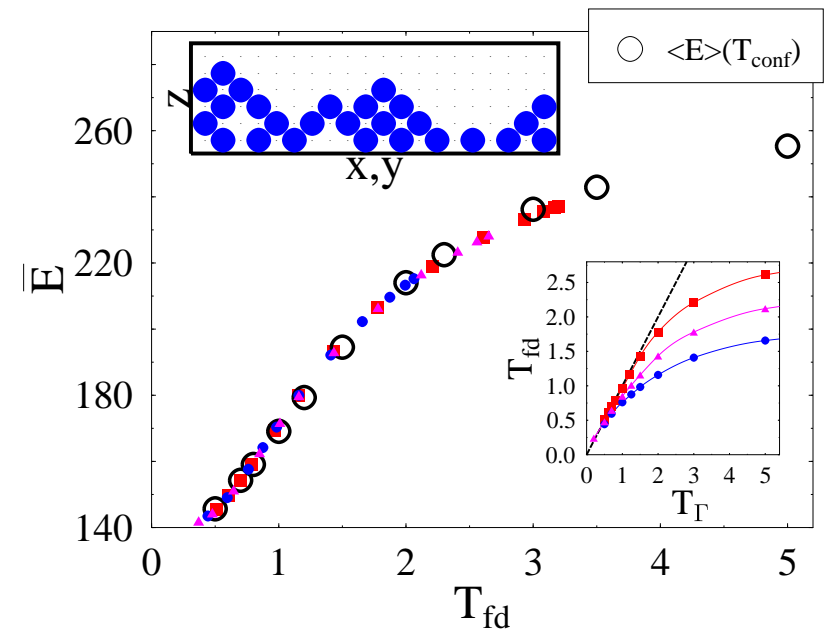

FIG. 11: Main frame The time average, $\bar{E}$, and the ensemble average over the distribution eq.(3), $\langle E\rangle$ (the black empty circles), plotted respectively as a function of $T_{f d}$ and $T_{\text {conf }}$ (in units $\left.m g a_{0}\right)$, in the 3D monodisperse hard-sphere system under gravity described in the text (and schematically depicted in the upper inset). Time averages over the tap dynamics and Edwards' ensemble averages then coincide. Lower Inset The temperature $T_{f d} \equiv \beta_{f d}^{-1}$ defined by eq.(2) as function of $T_{\Gamma}$ (in units $m g a_{0}$ ) for $\tau_{0}=500,10,5 M C S$ (from top to bottom). The straight line is the function $T_{f d}=T_{\Gamma}$.

overall Hamiltonian is:

$$
\mathcal{H}=\mathcal{H}_{h c}+m_{1} g H_{1}+m_{2} g H_{2}
$$

where $H_{1}=\sum_{i}^{(1)} z_{i}$ and $H_{2}=\sum_{i}^{(2)} z_{i}$, the height of site $i$ is $z_{i}$ and the two sums are over all particles of species 1 and 2 respectively. In the above units, the gravitational energies in a given configuration are thus $E_{1}=H_{1}$ and $E_{2}=2 H_{2}$.

Grains are confined in a box of linear size $L$ between hard walls and periodic boundary conditions in the horizontal directions. $N_{1}=120$ grains of type 1 and $N_{2}=40$ grains of type 2 are initially prepared in a random loose stable pack. Under the tap dynamics the system approaches a stationary state for each value of the tap parameters $T_{\Gamma}$ and $\tau_{0}$. In Fig.12, we plot as function of $T_{\Gamma}$ (for several values of $\tau_{0}$ ) the asymptotic value of the vertical segregation parameter, i.e., the difference of the average heights of the small and large grains at stationarity, $\Delta h\left(T_{\Gamma}, \tau_{0}\right) \equiv h_{1}-h_{2}$. Here $h_{1}$ and $h_{2}$ are the average of $H_{1} / N_{1}$ and $H_{2} / N_{2}$ over the tap dynamics in the stationary state. (An interpretation, in terms of the approach here presented, of the size segregation phenomenon here found and experimentally observed in a hard-spheres binary mixture under gravity is given in Ref. [15]).

The results given in the main panel of Fig. 12 apparently show that $T_{\Gamma}$ is not a right thermodynamic parameter, since sequences of taps with different $\tau_{0}$ give different values for the system observables. However, if

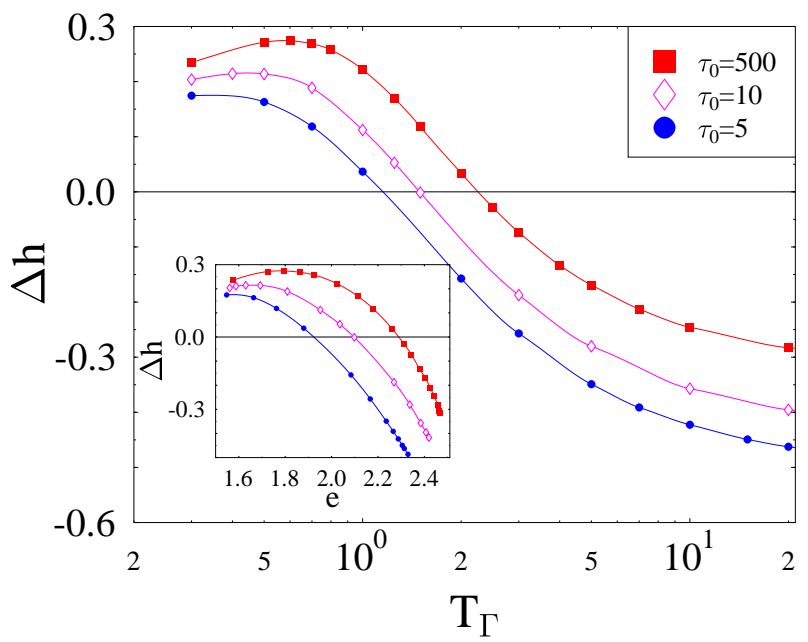

FIG. 12: Main frame The difference of the average heights of small and large grains, $\Delta h=h_{1}-h_{2}$, measured at stationarity in the binary hard spheres mixture under gravity, is plotted as a function of tap amplitude, $T_{\Gamma}$ (in units $m g a_{0}$ ). The three sets of points correspond to the shown tap durations, $\tau_{0}$. At high $T_{\Gamma}$ larger grains are found above the smaller, i.e, $\Delta h<0$, as in the Brazil nut effect (BNE). Below a $T_{\Gamma}^{*}\left(\tau_{0}\right)$ the opposite is found (Reverse Brazil nut effect, RBNE). Inset The $\Delta h$ data of the main frame are plotted as a function of the corresponding average energy, $e$. The three sets of data do not collapse onto a single master function, showing that a single macroscopic observable, such as $e$, doesn't characterize the system status.

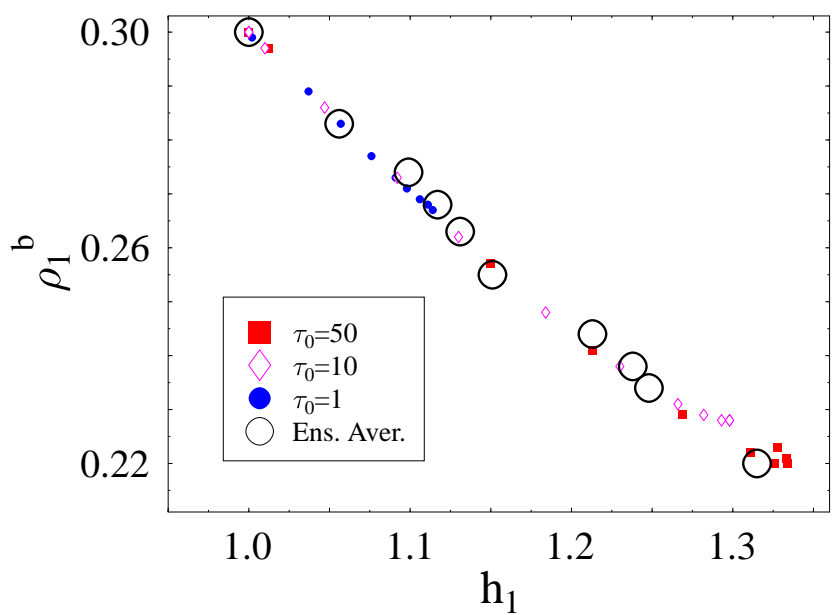

FIG. 13: The average density of small grains on the box bottom layer, $\rho_{1}^{b}$, measured at stationarity as a function of the height of small particles, $h_{1}$, in the binary hard spheres mixture under gravity. Data corresponding to different $T_{\Gamma}$ and $\tau_{0}$ approximately scale on a single master function. The empty circles are the corresponding values obtained by ensemble average with the two temperatures Gibbs measure proposed in the text. 


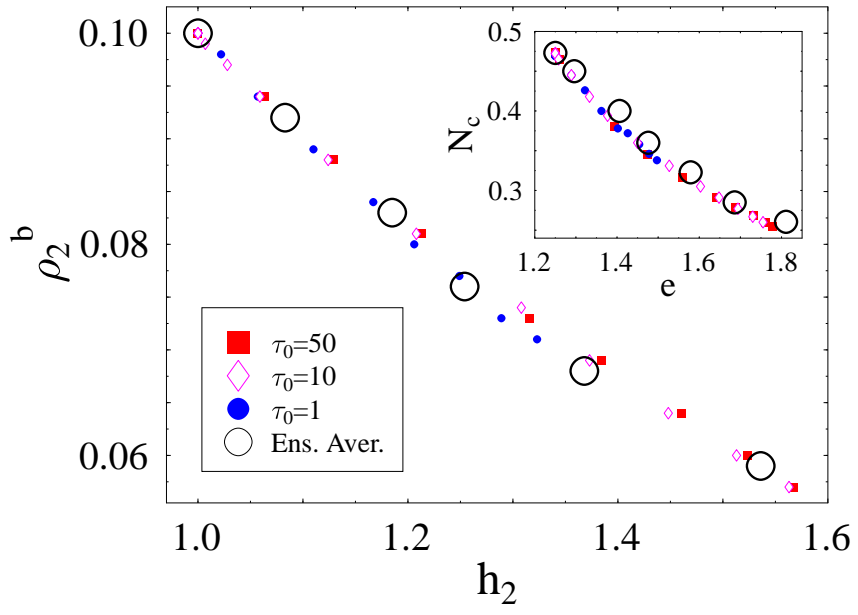

FIG. 14: Main frame The average density of large grains on the box bottom layer, $\rho_{2}^{b}$, obtained for different $T_{\Gamma}$ and $\tau_{0}$, scale almost on a single master function when plotted as a function of the large grains height, $h_{2}$. Upper inset The average number of contacts between large grains per particle, $N_{c}$, obtained for different $T_{\Gamma}$ and $\tau_{0}$, scale on a single master function when plotted as a function of the system energy, $e$.

the stationary states corresponding to different tap dynamics (i.e., different $T_{\Gamma}$ and $\tau_{0}$ ), are indeed characterized by a single thermodynamic parameter, the curves of Fig. 12 should collapse onto a universal master function when $\Delta h\left(T_{\Gamma}, \tau_{0}\right)$ is parametrically plotted as function of an other macroscopic observable such as the average energy, $e\left(T_{\Gamma}, \tau_{0}\right)=\left(E_{1}+E_{2}\right) / N$ ( $N$ is the total number of particles). This collapse of data is clearly not observed here, as it is apparent in the inset of Fig.12. We show, instead, that two macroscopic quantities may be sufficient to characterize uniquely the stationary state of the system. These two quantities are, for instance, the energy $e$ and the height difference $\Delta h$. Of course since $e=a h_{1}+2 b h_{2}$ (where $a=N_{1} / N$ and $b=N_{2} / N$ ) and $\Delta h=h_{1}-h_{2}$, we can also choose $h_{1}$ and $h_{2}$ to characterize the stationary state. Namely, we show that any macroscopic quantity $A$, averaged over the tap dynamics in the stationary state, is only dependent on $h_{1}$ and $h_{2}$, i.e., $A=A\left(h_{1}, h_{2}\right)$. We have checked that this is the case for several independent observables, such as the number of contacts between large particles, $N_{c}$, the density of small and large particles on the bottom layer, $\rho_{1}^{b}$ and $\rho_{2}^{b}$, and others. In particular, as shown in Fig.s 13 and 14, we find with good approximation that: $N_{c} \simeq N_{c}(e)=N_{c}\left(a h_{1}+b h_{2}\right), \rho_{2}^{b} \simeq \rho_{2}^{b}\left(h_{2}\right), \rho_{1}^{b} \simeq \rho_{1}^{b}\left(h_{1}\right)$. Therefore we need both $h_{1}$ and $h_{2}$ to characterize unambiguously the state of the system; namely all the observables assume the same values in a stationary state characterized by the same values of $h_{1}$ and $h_{2}$, independently on the previous history (i.e., in our case independently on the particular tapping parameters $T_{\Gamma}$ and $\tau_{0}$ ).

We again find that the stationary state can be genuinely considered as a thermodynamic state. Therefore we can ask what is the probability distribution, $P_{r}$, of finding the system in the inherent state $r$ corresponding to an energy $E_{1 r}$ for the small particles and $E_{2 r}$ for the large particles. We again assume that the microscopic distribution is given by the principle of maximum entropy $S=-\sum_{r} P_{r} \ln P_{r}$, now under the condition that the average energy $E_{1}=\sum_{r} P_{r} E_{1 r}$ and $E_{2}=\sum_{r} P_{r} E_{2 r}$ are independently fixed. This can be done by introducing two Lagrange multipliers $\beta_{1}$ and $\beta_{2}$, which are determined by the constraint on $E_{1}$ and $E_{2}$, and can be respectively considered as the "inverse configurational temperature" of species 1 and 2. This procedure leads to the Gibbs result:

$$
P_{r}=\frac{e^{-\beta_{1} E_{1 r}-\beta_{2} E_{2 r}}}{Z}
$$

where $Z=\sum_{r} \exp \left[-\beta_{1} E_{1 r}-\beta_{2} E_{2 r}\right]$ and, in the thermodynamic limit, the entropy, $S$, is given by:

$$
S=\ln \Omega\left(E_{1}, E_{2}\right)
$$

and $\beta_{1}$ and $\beta_{2}$ :

$$
\beta_{1}=\frac{\partial \ln \Omega\left(E_{1}, E_{2}\right)}{\partial E_{1}}, \quad \beta_{2}=\frac{\partial \ln \Omega\left(E_{1}, E_{2}\right)}{\partial E_{2}} .
$$

Here $\Omega\left(E_{1}, E_{2}\right)$ is the number of inherent states corresponding to energy $E_{1}$ and $E_{2}$. The hypothesis that the ensemble distribution at stationarity is given by eq.(7) can be tested as follows. We have to check that the time average of any quantity, $A\left(h_{1}, h_{2}\right)$, as recorded during the taps sequences in a stationary state characterized by given values $h_{1}$ and $h_{2}$, must coincide with the ensemble average, $\langle A\rangle\left(h_{1}, h_{2}\right)$, over the distribution eq.(7). To this aim, we have calculated the ensemble averages $\left\langle N_{c}\right\rangle,\left\langle\rho_{2}^{b}\right\rangle$, $\left\langle\rho_{1}^{b}\right\rangle$ for different values of $\beta_{1}$ and $\beta_{2}$; we have expressed parametrically $\left\langle N_{c}\right\rangle,\left\langle\rho_{2}^{b}\right\rangle,\left\langle\rho_{1}^{b}\right\rangle$, as function of the average of $h_{1}$ and $h_{2}$, and compared them with the corresponding quantities, $N_{c}, \rho_{1}^{b}$ and $\rho_{2}^{b}$, averaged over the tap dynamics. The two sets of data are plotted in Fig.s 13 and 14 showing a good agreement (notice, there are no adjustable parameters). In order to calculate the ensemble averages we simulate the model with $\mathcal{H}$ from eq.(6) where we impose the constraint that the only accessible states are the inherent states, as already described in Sect. II O.

\section{CONCLUSIONS}

In conclusion, in the context of models for glasses and granular materials we have shown that the stationary (or quasi-stationary) state reached by the system subject to a tap dynamics among its inherent states is genuinely a thermodynamic state, which can be well described by Edwards' assumption of an uniform measure, i.e., a probability distribution obtained assuming the validity of the principle of maximum entropy. In particular in the frustrated lattice gas model and in the system of monodisperse hard-spheres under gravity we have found that the 
observables recorded during different tap sequences (different amplitude and duration of taps) fall on universal master curves when plotted as a function of a single thermodynamic parameter. These curves turn out to coincide with those predicted, within the described Statistical Mechanics approach, by the generalized Gibbs distribution of eq.(3). On the other hand, the results obtained in a system under gravity made of particles of two different sizes show that a single thermodynamic parameter is not enough to describe the macrostates, and two configurational temperatures are instead necessary. In general, for a more complex system one might expect more constraints to be imposed, leading to more than two thermodynamic parameters 15, 18, 23. In practice, the criteria to determine a priori the required parameters can be not easily accessible. However more recently we have extended data regarding the hard-sphere binary mixture for very low energy [36] and found that only one thermodynamical parameter is necessary to describe the stationary state. This seems to be a general feature [14. If this is the case, a Statistical Mechanics approach with only one thermodynamical variable may be feasible for low energy.

\section{Acknowledgments}

This work was partially supported by the TMRERBFMRXCT980183, INFM-PRA(HOP), MURSTPRIN 2000 and MIUR-FIRB 2002. The allocation of computer resources from INFM Progetto Calcolo Parallelo is acknowledged.

\section{APPENDIX A: DETERMINATION OF THE INTEGRATION CONSTANT $\beta_{0}$}

Adapting to lattice models the procedure of Sciortino et al. [3], we have evaluated $\beta_{f d}$ at small values of $T_{\Gamma}$, for $\tau_{0} \rightarrow \infty$, and consequently the integration constant, $\beta_{0}$.

Given an inherent state, $r$, of energy, $E_{r}$, we define the basin of attraction, $B_{r}$, of such state $r$ as the set of states in the configurational space, which after the quench at $T=0$ are frozen in the inherent state $r$. Therefore the probability distribution, $P_{r}$, of finding the system in the inherent state $r$ after quenching the system from an equilibrium state at temperature $T_{\Gamma}$, can be written as

$$
P_{r}=\frac{\sum_{r^{\prime}} e^{-E_{r r^{\prime}} / T_{\Gamma}}}{Z_{G}\left(T_{\Gamma}\right)}
$$

where $Z_{G}\left(T_{\Gamma}\right)$ is the partition function of the system in equilibrium at temperature $T_{\Gamma}$ and $\sum_{r^{\prime}}$ is the the sum over all the states $r^{\prime}$ belonging to the basin $B_{r}$ of energy $E_{r r^{\prime}}$. By putting $E_{r r^{\prime}}=E_{r}+\Delta_{r r^{\prime}}$, the distribution (A1) can be written as

$$
P_{r}=\frac{e^{-\left(E_{r}+g_{r}\left(T_{\Gamma}\right)\right) / T_{\Gamma}}}{Z_{G}\left(T_{\Gamma}\right)},
$$

where $e^{-g_{r}\left(T_{\Gamma}\right) / T_{\Gamma}}=\sum_{r^{\prime}} e^{-Z_{G r^{\prime}}\left(T_{\Gamma}\right)} T_{\Gamma}$. From eq. (A2) it follows that the probability of finding the system in any inherent state of energy $E, P\left(E, T_{\Gamma}\right)=\sum_{r} P_{r}$ (where $\sum_{r}$ is the sum over all the inherent states $r$ of energy $E$ ) is given by

$$
P\left(E, T_{\Gamma}\right)=\frac{\Omega(E) e^{-E / T_{\Gamma}} e^{-f\left(T_{\Gamma}, E\right) / T_{\Gamma}}}{Z_{G}\left(T_{\Gamma}\right)},
$$

where $\Omega(E)$ is the number of inherent states of energy $E$ and $e^{-f\left(T_{\Gamma}, E\right) / T_{\Gamma}}=\frac{\sum_{r} e^{-g_{r}\left(T_{\Gamma}\right)} / T_{\Gamma}}{\Omega(E)}$. From eq. (A3):

$\ln \left[P\left(E, T_{\Gamma}\right)\right]+\frac{E}{T_{\Gamma}}=-\frac{f\left(T_{\Gamma}, E\right)}{T_{\Gamma}}+\ln [\Omega(E)]-\ln \left[Z_{G}\left(T_{\Gamma}\right)\right]$.

The probability distribution of finding the system in any inherent state of energy $E, P\left(E, T_{\Gamma}\right)$, is measured during the tap dynamics with amplitude $\tau_{0} \rightarrow \infty$. If $f\left(T_{\Gamma}, E\right)$ has only a weak dependence on $E$, then it is possible to superimposed the curves, $\ln \left[P\left(E, T_{\Gamma}\right)\right]+E / T_{\Gamma}$, at different $T_{\Gamma}$ which overlap in $E$ by adding a $T_{\Gamma}$-dependent constant. This result is obtained for $T_{\Gamma} \leq 0.525$, as shown in Fig.15, and suggests that in this interval $f\left(T_{\Gamma}, E\right) \simeq f\left(T_{\Gamma}\right)$. If this is the case, from eq. (A3) it follows:

$$
P\left(E, T_{\Gamma}\right) \simeq \frac{\Omega(E) e^{-E / T_{\Gamma}}}{Z\left(T_{\Gamma}\right)}
$$

where

$$
Z\left(T_{\Gamma}\right)=e^{f\left(T_{\Gamma}\right) / T_{\Gamma}} Z_{G}\left(T_{\Gamma}\right)=\sum_{E} \Omega(E) e^{-E / T_{\Gamma}} .
$$

The last equality stems from the normalization condition on $P\left(E, T_{\Gamma}\right)$.

From eq. A5 it follows that at small $T_{\Gamma}, \beta_{\Gamma} \equiv T_{\Gamma}^{-1}$ satisfies eq.(2). Therefore at small $T_{\Gamma}, \beta_{f d}$ and $\beta_{\Gamma}$ coincide. The constant $\beta_{0}$ is consequently obtained as the limit, for $T_{\Gamma} \rightarrow 0$, of the function $\beta_{\Gamma}-g\left(\beta_{\Gamma}\right)$ (see Fig.16).
[1] F.H. Stillinger and T.A. Weber, Phys. Rev. A 25, 978 (1982); Science 225, 983 (1984). F. H. Stillinger, Science, 267 1935, (1995). S. Sastry, P.G. Debenedetti, F.H. Stillinger, Nature 393, 554 (1998).
[2] B. Coluzzi, G. Parisi and P. Verrocchio, Phys. Rev. Lett. 84, 306 (2000).

[3] F. Sciortino, W. Kob, P. Tartaglia, Phys. Rev. Lett. 83, 3214 (1999). 


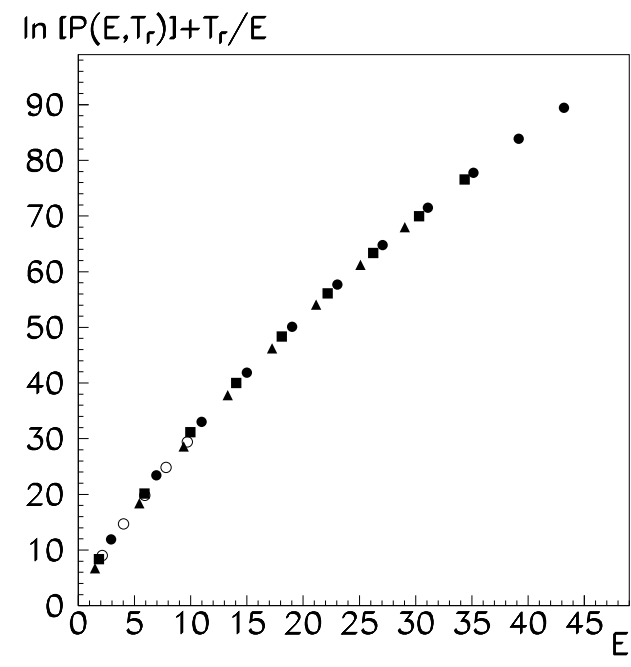

FIG. 15: The curves $\ln \left[P\left(E, T_{\Gamma}\right)\right]+E / T_{\Gamma}$ (apart from a $T_{\Gamma}$-dependent constant) as function of the energy $E$ in the frustrated lattice gas model for $\rho=0.65$ and $T_{\Gamma}=$ $0.275,0.425,0.475,0.525 \mathrm{~J}$.

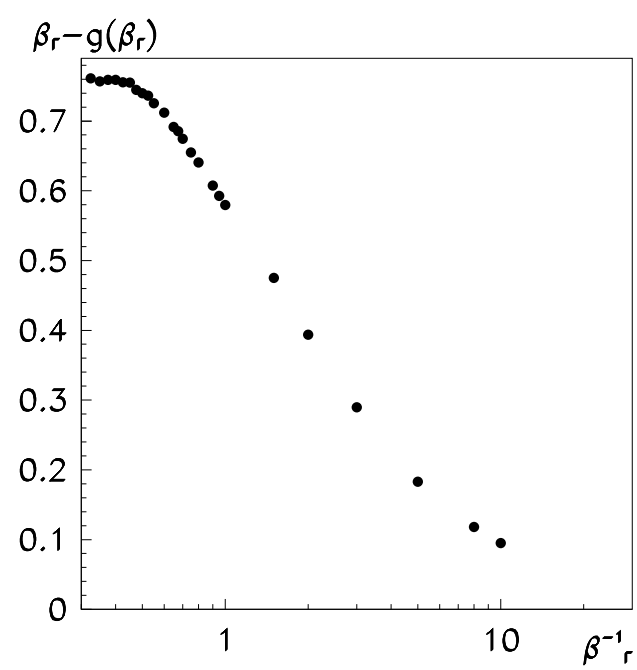

FIG. 16: The curve $\beta_{\Gamma}-g\left(\beta_{\Gamma}\right)=\beta_{\Gamma}-\left[\beta_{f d}\left(\beta_{\Gamma}\right)-\beta_{0}\right]$ as function of $\beta_{\Gamma}^{-1}$ (in units of $J$ ) in the frustrated lattice gas model for $\rho=0.65$. The limit $\beta_{\Gamma}^{-1} \rightarrow 0$ of $\beta_{\Gamma}-g\left(\beta_{\Gamma}\right)$ gives the integration constant $\beta_{0}$.

[4] W. Kob, F. Sciortino and P. Tartaglia, Europhys. Lett., 49, 590 (2000).

F. Sciortino and P. Tartaglia, Phys. Rev. Lett. 86, 107 (2001).

[5] H.M. Jaeger, S.R. Nagel and R.P. Behringer, Rev. Mod. Phys. 68, 1259 (1996).

[6] Grains are "frozen" because, due to their large masses and dissipation [5], the thermal kinetic energy is negligible compared to the gravitational energy; thus the external bath temperature, $T_{b a t h}$, can be considered equal to zero.

[7] S.F. Edwards and R.B.S. Oakeshott, Physica A 157,
1080 (1989). A. Mehta and S.F. Edwards, Physica A 157 1091 (1989). S.F. Edwards, in "Disorder in Condensed Matter Physics" page. 148, Oxford Science Pubs (1991); and in Granular Matter: an interdisciplinary approach, (Springer-Verlag, New York, 1994), A. Mehta ed.

[8] L. F. Cugliandolo, J. Kurchan and L. Peliti, Phys. Rev. E 55,3898 (1997).

[9] R. Monasson, Phys. Rev. Lett. 75, 2847 (1995). Th.M. Nieuwenhuizen, Phys. Rev. E 61, 267 (2000). S. Franz and M.A. Virasoro, J. Phys. A 33, 891 (2000). A. Crisanti and F. Ritort, Journal of Chemical Physics 113, 10615 (2000). J. Kurchan, cond-mat/981234"; and in "Jamming and Rheology: Constrained Dynamics on Microscopic and Macroscopic Scales", A.J. Liu and S.R. Nagel Eds., Taylor and Francis, London (2001).

[10] M. Nicodemi, Phys. Rev. Lett. 82, 3734 (1999).

[11] A. Barrat, J. Kurchan, V. Loreto and M. Sellitto, Phys. Rev. Lett. 85, 5034 (2000); Phys. Rev. E 63,051301 (2001)

[12] H. A. Makse and J. Kurchan, Nature 415, 614 (2002).

[13] A. Coniglio and M. Nicodemi, Physica A 296, 451 (2001).

[14] A. Coniglio, A. Fierro and M. Nicodemi, Physica A 302 (1-4): 193 (2001); A. Fierro, M. Nicodemi and A. Coniglio, Europhys. Lett. 59, 642 (2002); A. Coniglio, A. Fierro and M. Nicodemi, Europ. Phys. Jour. E, in press (2002).

[15] M. Nicodemi, A. Fierro and A. Coniglio, condmat/0202500 and Europhys. Lett. in press.

[16] J.J. Brey, A. Prados, B. Sánchez-Rey, Physica A 275, 310 (2000); A. Prados, J.J. Brey, B. Sánchez-Rey, Physica A 284, 277 (2000).

[17] D. S. Dean and A. Lefèvre, Phys. Rev. Lett. 86, 5639 (2001); J. Phys. A: Math. Gen. 34, L213 (2001); condmat/0106220.

[18] J. Berg, S. Franz and M. Sellitto, Eur. Phys. J. B 26, 349 (2002).

[19] M. Nicodemi, A. Coniglio, H.J. Herrmann, Phys. Rev. E 55, 3962 (1997); J. Phys. A 30, L379 (1997). A. Coniglio and H.J. Herrmann, Physica A 225, 1 (1996).

[20] J. Berg, A. Mehta, Europhys. Lett. 56, 784 (2001).

[21] L. Berthier, L.F. Cugliandolo and J.L. Iguain, Phys. Rev. E 63, 051302 (2001).

[22] the possibility of introducing more than one thermodynamic parameter has been also suggested in 18 and recently discussed in the context of a Constrained Ising Chain in 23].

[23] A. Lefèvre, cond-mat/0202376.

[24] A. Mehta and G. C. Barker, Phys. Rev. Lett. 67, 394 (1991).

[25] A. Coniglio, A. de Candia, A. Fierro, M. Nicodemi, Jour. Phys.: Cond. Mat. 11, A167 (1999).

[26] A. Coniglio, "Frustration and connectivity in glass forming systems and granular materials", in Proc. Int. School on the Physics of Complex Systems "Enrico Fermi" CXXXIV course (Varenna 1996), edited by F. Mallamace and H.E. Stanley (IOS Press, Amsterdam,1997), p.491. M. Nicodemi and A. Coniglio, J. Phys A Lett. 30, L187 (1996). F. Ricci-Tersenghi, D.A. Stariolo and J.J. Arenzon, Phys. Rev. Lett. 84, 4473 (2000).

[27] A. Coniglio, M. Nicodemi, J. Phys.: Cond. Matt. 12, 6601 (2000); M. Nicodemi, A. Coniglio, Phys. Rev. Lett. 82, 916 (1999).

[28] J.J. Arenzon, J. Phys. A. 32, L107 (1999).

[29] A. de Candia and A. Coniglio, Phys. Rev. E 65, 16132 
(2002).

[30] $\tau_{0}$ is measured in Monte Carlo steps (MCS), where $1 M C S$ corresponds to $N$ attempts to move a particle randomly chosen, and $N=\sum_{i} n_{i}$ is the number of particles.

[31] Note that with our approach it is also possible to explore low density inherent states in a stationary regime and not only the off-equilibrium "glassy regime", as instead in Ref. 11. For instance, the frustrated lattice gas model at density $\rho=0.65$ is hardly found in an out of equilibrium quasi-stationary state (at any finite value of the bath temperature the system quickly reaches the equilibrium state). We have considered the model eq.(伍), at $\rho=0.65$, and we have performed an usual Monte Carlo diffusive dynamics of the model. At a starting time we prepared the system in equilibrium with a very high bath temperature. Afterwards, it is suddenly cooled at a very low $T_{\text {bath }}$, but the system quickly reaches the equilibrium state, and $T_{d y n}$ coincides with $T_{b a t h}$.
[32] Where not explicitely shown, the error bars of the data are more or less equal to the size of symbols in figures.

[33] J.B. Knight, C.G. Fandrich, C.N. Lau, H.M. Jaeger and S.R. Nagel, Phys. Rev. E 51, 3957 (1995). E.R. Nowak, J.B. Knight, E. Ben-Naim, H.M. Jaeger and S.R. Nagel, Phys. Rev. E 57, 1971 (1998). E.R. Nowak, J.B. Knight, M. Povinelli, H.M. Jaeger, and S.R. Nagel, Powder Technology 94, 79 (1997).

[34] In both cases, $\rho=0.65$ and 0.75 , the stationary self scattering functions obtained for finite tap duration $\tau_{0}$, are well fitted by stretched exponentials, $e^{-(t / \tau)^{a}}$. The characteristic time scale, $\tau$, obtained in this way increases as $T_{\Gamma}$ decreases, and seems to diverge only in the limit $T_{\Gamma} \rightarrow 0$.

[35] In the previous case, $\rho=0.65$, the minimum energy, $E_{\min }$, is zero.

[36] A. Coniglio, A. Fierro, and M. Nicodemi (unpublished). 